\title{
Bleaching and diffusion dynamics in optofluidic dye lasers
}

\author{
Gersborg-Hansen, Morten; Balslev, Søren; Mortensen, Asger; Kristensen, Anders
}

Published in:

Applied Physics Letters

Link to article, DOI:

$10.1063 / 1.2718503$

Publication date:

2007

Document Version

Publisher's PDF, also known as Version of record

Link back to DTU Orbit

Citation (APA):

Gersborg-Hansen, M., Balslev, S., Mortensen, A., \& Kristensen, A. (2007). Bleaching and diffusion dynamics in optofluidic dye lasers. Applied Physics Letters, 90(14), 143501. https://doi.org/10.1063/1.2718503

\section{General rights}

Copyright and moral rights for the publications made accessible in the public portal are retained by the authors and/or other copyright owners and it is a condition of accessing publications that users recognise and abide by the legal requirements associated with these rights.

- Users may download and print one copy of any publication from the public portal for the purpose of private study or research.

- You may not further distribute the material or use it for any profit-making activity or commercial gain

- You may freely distribute the URL identifying the publication in the public portal

If you believe that this document breaches copyright please contact us providing details, and we will remove access to the work immediately and investigate your claim. 


\title{
Bleaching and diffusion dynamics in optofluidic dye lasers
}

\author{
Morten Gersborg-Hansen, Søren Balslev, ${ }^{\text {a) }}$ \\ Niels Asger Mortensen, and Anders Kristensen ${ }^{\text {b) }}$ \\ MIC-Department of Micro and Nanotechnology, NanoDTU, Technical University of Denmark, \\ Building 345east, Ørsteds Plads, DK-2800 Kongens Lyngby, Denmark
}

(Received 2 February 2007; accepted 25 February 2007; published online 2 April 2007)

\begin{abstract}
The authors have investigated the bleaching dynamics that occur in optofluidic dye lasers where the liquid laser dye in a microfluidic channel is locally bleached due to optical pumping. They find that for microfluidic devices, the dye bleaching may be compensated through diffusion of dye molecules alone. By relying on diffusion rather than convection to generate the necessary dye replenishment, their observation potentially allows for a significant simplification of optofluidic dye laser device layouts, omitting the need for cumbersome and costly external fluidic handling or on-chip microfluidic pumping devices. @ 2007 American Institute of Physics. [DOI: 10.1063/1.2718503]
\end{abstract}

Optical techniques have proven powerful in chemical and biochemical analysis. This has stimulated a large effort in integrating fluidics and optics in lab-on-a-chip microsystems, ${ }^{1}$ partly defining the emerging field of optofluidics, as recently reviewed by Psaltis et al. ${ }^{2}$ and Monat $e t \mathrm{al}^{3}$ Among the investigated components are miniaturized fluidic dye lasers, also referred to as optofluidic dye lasers. ${ }^{4-9}$ The so far reported optofluidic dye lasers are pulsed in order to have a short interaction time between the dye molecules and the pump light, thus suppressing the formation of triplet states unsuitable for lasing. This contrasts macroscopic continuous-wave dye lasers where the suppression is mediated by a jet flow of the dye solution with typical velocities of several $\mathrm{m} / \mathrm{s}^{10}$

Dye bleaching resulting in limited lifetime is in general considered a major disadvantage of organic dyes as active laser medium. Typically, the problem of dye bleaching is addressed by employing a continuous convective flow of liquid-dissolved dye molecules, compensating the bleaching dynamics caused by the external optical pump. In optofluidic dye lasers, the required convective dye-replenishing flow has been achieved by external fluid-handling apparatus (syringe pumps), ${ }^{4-8}$ an on-chip microfluidic pump, ${ }^{11}$ or by means of capillary effect. ${ }^{9,12}$

In this letter we demonstrate that such optofluidic devices may potentially be operated for days by diffusion without the need for a convective flow. The key concept in this is very similar to the entire paradigm behind miniaturized chemical-analysis systems where scaling arguments are used to show the attractiveness of micron-scale analytical devices compared to their macroscopic counterparts, see Janasek et $a l .{ }^{13}$ for a recent review.

We have investigated the local dye bleaching dynamics which is characteristic in optofluidic dye lasers. Based on our findings we propose a dye replenishment mechanism which takes advantage of the classical diffusion present when dye molecules are dissolved in a liquid and placed in a microfluidic device. The bleaching of dye molecules by the optical pump will introduce gradients in the concentration of non-

\footnotetext{
a) Present address: Ignis Photonyx A/S, Blokken 84, DK-3460 Birkerød, Denmark.

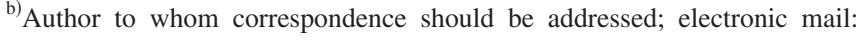
ak@mic.dtu.dk; URL: www.optofluidics.dk and www.mic.dtu.dk/nil
}

bleached dye molecules $c(\mathbf{r}, t)$, thus activating the diffusion mechanism associated with the thermally driven Brownian motion of the dye molecules. The diffusion-bleaching dynamics is governed by a classical diffusion-convection equation with an additional drain (sink) term

$$
D \nabla^{2} c(\mathbf{r}, t)=\frac{\partial}{\partial t} c(\mathbf{r}, t)+\mathbf{v} \cdot \nabla c(\mathbf{r}, t)+\Gamma(\mathbf{r}) c(\mathbf{r}, t),
$$

where $\Gamma$ is the bleaching rate, $D$ is the diffusion constant, and $\mathbf{v}$ is the velocity field of a possible flow. For rhodamine $6 \mathrm{G}$ dye molecules dissolved in ethylene glycol, $D$ is estimated to $D \sim 1.5 \times 10^{-11} \mathrm{~m}^{2} / \mathrm{s}$ by taking an experimental value for rhodamine $6 \mathrm{G}$ molecules in water ${ }^{14}$ and scaling with the viscosity at $25{ }^{\circ} \mathrm{C}$, using the Stokes-Einstein relation. On average, the random walk transports a dye molecule approximately $0.1 \mathrm{~mm}$ in $10 \mathrm{~min}$. For typical optical pumping levels and repetition rates we estimate this to be sufficient to replenish bleached dye in a miniaturized dye laser. This statement is supported by both Eq. (1) and by our experimental studies of a particular optofluidic device.

To analyze the bleaching-diffusion dynamics, we use a simple, idealized one-dimensional model system, resembling the experimental setup outlined in Fig. 1. We consider a situ-

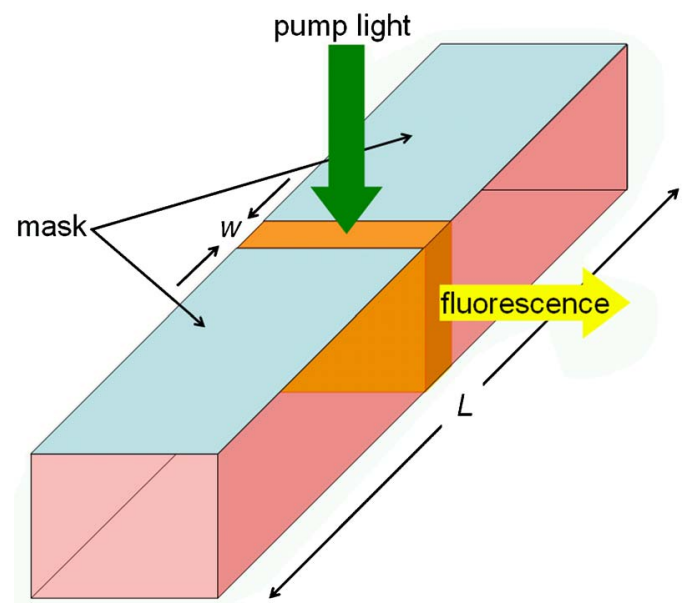

FIG. 1. Experimental setup. Closed channel containing a liquid solution of dye molecules. The dye molecules are optically pumped by a pulsed, frequency-doubled Nd:YAG laser through a slit of width $w$ covering the sample. 
ation where the liquid-dissolved dye molecules are optically pumped through a narrow slit of width $w$ covering a microfluidic channel of length $L$ connecting two ideal liquid reservoirs supporting an always constant concentration $c_{0}$ (not shown in the figure). With $x$ being along the direction of the quasi-one-dimensional channel we can thus make the approximation $\Gamma(x) \simeq \Gamma_{0} \Theta(w / 2-|x|)$, where $\Theta(x)$ is the Heaviside step function with $\Theta(x)=0$ for $x<0$ and $\Theta(x)=1$ for $x>0$. In the symmetric case the concentration will of course always have a minimum in the center of the slit so in the following we focus on $x=0$ and suppress the spatial variable for the sake of clarity.

The dynamics may conveniently be understood by dimensional analysis. Since the characteristic time scale is set by the inverse bleaching rate $\Gamma_{0}^{-1}$ and the characteristic length scale by the slit width $w$, we readily arrive at a diffusion rate $\Gamma_{D}=D / w^{2}$ and a convection rate $\Gamma_{v}=v / w$ which should be compared to the externally controlled bleaching rate $\Gamma_{0}$. In macroscopic dye lasers one has $\Gamma_{v} \gg \Gamma_{0} \gg \Gamma_{D}$ so that unbleached dye molecules are supplied on a faster time scale than the bleaching. This strategy has been central to the so far reported microfluidic dye lasers. ${ }^{4-9,11,12}$ However, the very different scaling of $\Gamma_{D}$ and $\Gamma_{v}$ with $w$ offers an alternative and attractive replenishment mechanism in micron-scale systems. Usually the convective term $\mathbf{v} \cdot \nabla c$ is driving the replenishment, but if $\Gamma_{D} \gg \max \left(\Gamma_{v}, \Gamma_{0}\right)$ we have the freedom to completely turn off convection and entirely rely on diffusion. In this work we will emphasize diffusion and the convective term will be explicitly zero, i.e., $\mathbf{v}=0$.

The initial dynamics is characterized by the absence of gradients and for $t \ll \Gamma_{D}^{-1}$, we get the expected solution $c(t) \simeq c_{0} \exp \left[-\Gamma_{0} t\right]$. This exponential dynamics, with a characteristic time scale $\Gamma_{0}^{-1}$, is the major source of concern for the bleaching-defined lifetime of the device outlined in Fig. 1. However, the bleaching, expressed as $c \rightarrow 0$ for $t \gg \Gamma_{0}^{-1}$, may be compensated by diffusion which becomes efficient for $t \gtrsim \Gamma_{D}^{-1}$. The addition of rates (like Matthiessen's rule for addition of scattering rates) suggests that for $t \geqslant \Gamma_{D}^{-1}$ the decay will be governed by $\Gamma_{D}$ rather than $\Gamma_{0}$. Equation (1) is difficult to solve analytically, but the dynamics may easily be studied numerically in more detail. Figure 2 illustrates the bleaching-diffusion dynamics for a channel much longer than the slit width, $L \gg w$. As seen, the above analysis accounts well for the qualitative behavior found from timedependent finite-element simulations of Eq. (1) for varying values of $\Gamma_{D}$ relative to $\Gamma_{0}$. Clearly, diffusion changes the dynamics away from the pure exponential bleaching (lower curve) to a situation with a much slower decay, thus potentially increasing the bleaching-limited lifetime of the device dramatically.

Earlier experiments on dye-doped polymer lasers suggest that the bleaching rate is of the order $\Gamma_{0} \sim 10^{-4} \mathrm{~s}^{-1}$ for typical concentrations and pumping levels. ${ }^{15}$ The diffusion rate $\Gamma_{D}$ would be comparable to $\Gamma_{0}$ for a slit of macroscopic width $w \sim 1 \mathrm{~mm}$. For a micron-scale slit of width $w \sim 100 \mu \mathrm{m}$, however, the diffusion rate increases by one order of magnitude to $\Gamma_{D} \sim 10^{-3} \mathrm{~s}^{-1}$. These order-ofmagnitude estimates illustrate the importance of the $w^{-2}$ scaling of $\Gamma_{D}$ and support our proposal of miniaturized optofluidic dye lasers with $\Gamma_{D} \gg \Gamma_{0}$, thus fully avoiding any need for external fluidic handling systems such as syringe pumps or complicated on-chip pumping schemes.

Downloaded 17 Jun 2010 to 192.38.67.112. Redistribution subject to AIP license or copyright; see http://apl.aip.org/apl/copyright.jsp

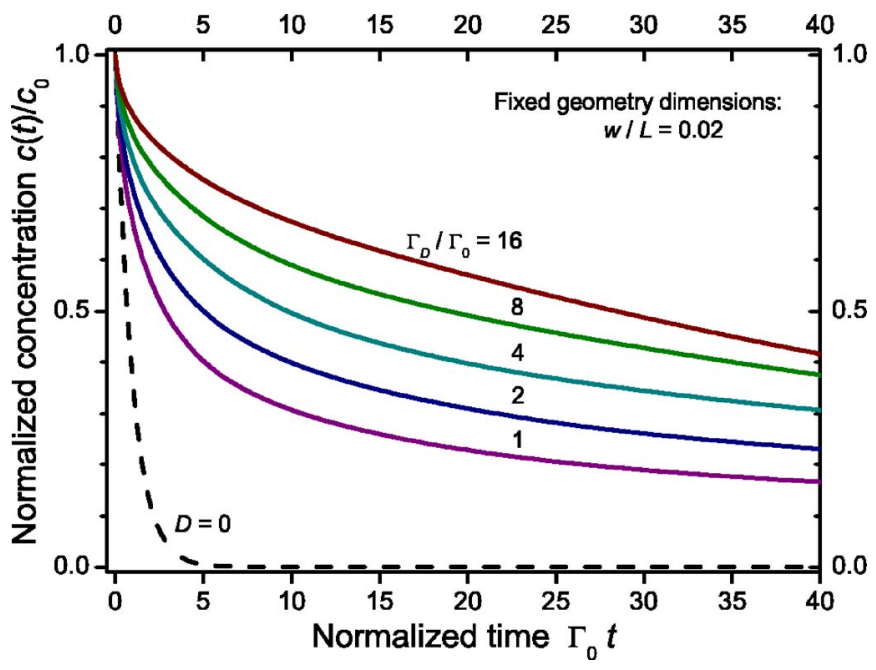

FIG. 2. Plot of dye molecule concentration vs time in a quasi onedimensional microfluidic channel of length $L$, see Fig. 1. Bleaching by the optical pump occurs through a narrow slit of width $w=0.02 L$ with a bleaching rate $\Gamma_{0}$. Diffusion changes the dynamics away from the pure exponential bleaching $(D=0)$ and yields a much slower decay.

The implicit hypothesis behind the above analysis is of course that the dye laser output signal somehow correlates with the concentration of unbleached dye molecules. In order to verify our predictions experimentally we use the intensity of the fluorescence as an indirect measure of the local dye concentration in the optically pumped volume. In the experiments, we filled a flexible polymer tube of diameter $\simeq 800 \mu \mathrm{m}$ with a $2 \times 10^{-4} \mathrm{~mol} / 1$ solution of rhodamine $6 \mathrm{G}$ in ethylene glycol. For this dye concentration level, the quantum efficiency has a weak dependency on the dye concentration. ${ }^{16}$ The liquid volume was confined to a length of $L=8 \mathrm{~mm}$ and the dye was locally pumped through a slit of varying width $w$ using a pulsed, frequency doubled Nd:YAG (yttrium aluminum garnet) laser: wavelength $532 \mathrm{~nm}$, pulse duration $5 \mathrm{~ns}$, repetition rate $10 \mathrm{~Hz}$, and average pulse energy fluence $32 \mu \mathrm{J} / \mathrm{mm}^{2}$. The chosen pumping level is typical for optofluidic dye lasers operating well above threshold. ${ }^{8,9,12}$ The fluorescence signal indicated in Fig. 1 was collected at an angle normal to the incident pump light by an optical fiber and measured by a fixed grating spectrometer (resolution $0.15 \mathrm{~nm}$ ).

Figure 3 shows the temporal decay of the integrated spectrally broad fluorescence signal for different optical pumping configurations. For spatially homogeneous optical pumping (no slit, $w / L=1$ ), the data show an initial fast decay, followed by a much slower decay with a characteristic decay time of $5.0 \mathrm{~h}$. The behavior of an initial fast decay followed by a slow decay does not arise from pump laser fluctuations or from changes in the spectral output pattern of the device. When pumping through a slit $(w / L<1)$, the fast decay remains and the rate of the slow decay decreases with $w$. In the case of $w / L=0.04(w \simeq 300 \mu \mathrm{m})$, a nearly stable output level on the time scale of days is observed. The experimentally observed fluorescence dynamics is in qualitative agreement with the bleaching-diffusion dynamics found in our quasi one-dimensional model, see Fig. 2. We would like to emphasize that such optofluidic devices provide a typical level of output power that is more than sufficient for use as an on-chip light source in lab-on-a-chip applications. ${ }^{17}$ 


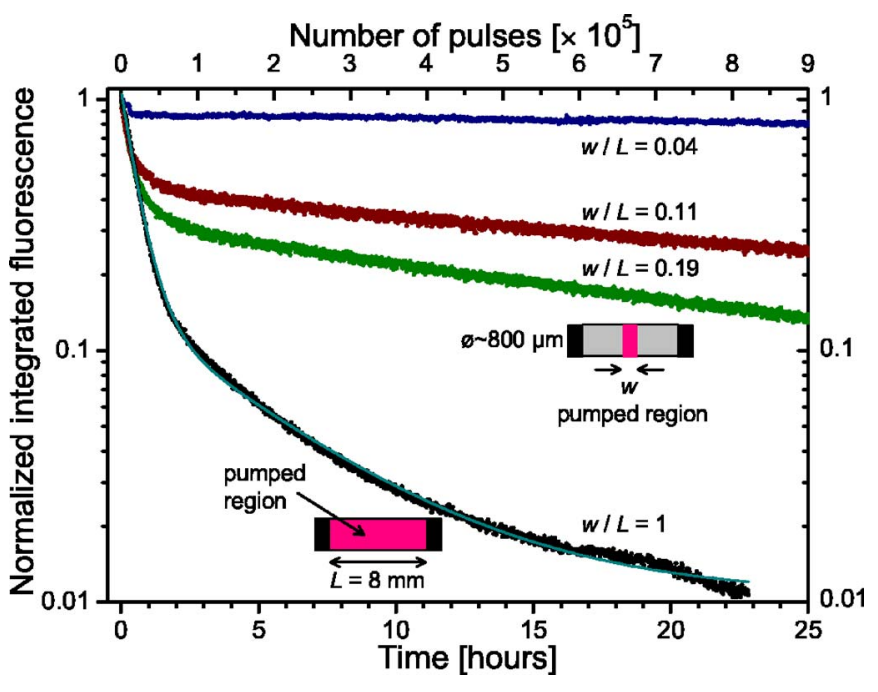

FIG. 3. Temporal decay of fluorescence for different optical pumping configurations. The lower curve $(w / L=1)$ is for spatially homogeneous pumping while the upper curves correspond to spatially inhomogeneous pumping through slits of varying width $w$. In the case of $w / L=0.04$, the fluorescence signal is nearly constant on the time scale of days after a short initial decay (first $25 \mathrm{~h}$ shown).

In conclusion, we have investigated the bleaching and diffusion dynamics in optofluidic dye lasers caused by local bleaching of the laser dye. A simple one-dimensional diffusion model was used to explore the characteristic evolution of the local unbleached dye concentration in the optically pumped or bleached volume of the device. In the absence of convective flow, the asymptotic decay of the local dye concentration in the optically pumped volume is governed by the diffusion rate and the resulting lifetime of the device is mainly limited by the capacity of the fluidic reservoirs. Generic microfluidic platforms typically allow for device layouts with a large volume ratio between the fluidic reservoir and the region being optically pumped. In order to put our proposal in perspective, a reservoir with a volume of the order $1 \mathrm{~cm}^{3}$, with an optically pumped volume of 2.5 $\times 10^{-7} \mathrm{~cm}^{3}$, and a bleaching rate of $\Gamma_{0} \sim 10^{-4} \mathrm{~s}^{-1},{ }^{15}$ would in principle allow for continuous operation for more than a thousand years for typical pumping levels and repetition rates. These conclusions drawn from the simple model are supported by basic experiments. Our investigations reveal the possibility that such optofluidic dye laser devices may potentially be operated for days by diffusion without the need for a convective flow. Relying on diffusion rather than convection to generate the necessary dye replenishment significantly simplifies optofluidic dye laser device layouts, omitting the need for cumbersome and costly external fluidic handling or on-chip microfluidic pumping devices.

This work was supported by the Danish Technical Research Council (STVF, Grant No. 26-02-0064) and the Danish Council for Strategic Research through the Strategic Program for Young Researchers (Grant No. 2117-05-0037).

${ }^{1}$ E. Verpoorte, Lab Chip 3, 42N (2003).

${ }^{2}$ D. Psaltis, S. R. Quake, and C. H. Yang, Nature (London) 442, 381 (2006).

${ }^{3}$ C. Monat, P. Domachuk, and B. J. Eggleton, Nat. Photon. 1, 106 (2007).

${ }^{4}$ B. Helbo, A. Kristensen, and A. Menon, J. Micromech. Microeng. 13, 307 (2003).

${ }^{5}$ S. Balslev and A. Kristensen, Opt. Express 13, 344 (2005).

${ }^{6}$ M. Gersborg-Hansen, S. Balslev, N. A. Mortensen, and A. Kristensen, Microelectron. Eng. 78-79, 185 (2005).

${ }^{7}$ D. V. Vezenov, B. T. Mayers, R. S. Conroy, G. M. Whitesides, P. T. Snee, Y. Chan, D. G. Nocera, and M. G. Bawendi, J. Am. Chem. Soc. 127, 8952 (2005).

${ }^{8}$ Z. Y. Li, Z. Y. Zhang, T. Emery, A. Scherer, and D. Psaltis, Opt. Express 14, 696 (2006).

${ }^{9}$ M. Gersborg-Hansen and A. Kristensen, Appl. Phys. Lett. 89, 103518 (2006).

${ }^{10}$ Dye Lasers, 3rd ed., edited by F. P. Schäfer (Springer, Berlin, 1990).

${ }^{11}$ J. C. Galas, J. Torres, M. Belotti, Q. Kou, and Y. Chen, Appl. Phys. Lett. 86, 264101 (2005).

${ }^{12}$ M. Gersborg-Hansen and A. Kristensen, Opt. Express 15, 137 (2007).

${ }^{13}$ D. Janasek, J. Franzke, and A. Manz, Nature (London) 442, 374 (2006).

${ }^{14}$ R. Rigler, Ü. Mets, J. Widengren, and P. Kask, Eur. Biophys. J. 22, 169 (1993).

${ }^{15}$ S. Balslev, T. Rasmussen, P. Shi, and A. Kristensen, J. Micromech. Microeng. 15, 2456 (2005).

${ }^{16}$ F. L. Arbeloa, P. R. Ojeda, and I. L. Arbeloa, J. Photochem. Photobiol., A 45, 313 (1988).

${ }^{17}$ S. Balslev, A. M. Jorgensen, B. Bilenberg, K. B. Mogensen, D. Snakenborg, O. Geschke, J. P. Kutter, and A. Kristensen, Lab Chip 6, 213 (2006). 